\title{
Inverse Source on Conformal Conic Geometries
}

This paper was downloaded from TechRxiv (https://www.techrxiv.org).

\section{LICENSE}

CC BY 4.0

SUBMISSION DATE / POSTED DATE

21-07-2020 / 02-10-2020

\section{CITATION}

Leone, Giovanni; Munno, Fortuna; Pierri, Rocco (2020): Inverse Source on Conformal Conic Geometries.

TechRxiv. Preprint. https://doi.org/10.36227/techrxiv.12682079.v2

$\mathrm{DOI}$

10.36227/techrxiv.12682079.v2 


\title{
Inverse Source on Conformal Conic Geometries
}

\author{
G. Leone, F. Munno, and R. Pierri
}

\begin{abstract}
The inverse source problem has a number of applications in antenna analysis and synthesis. The properties of the radiation operator, connecting the source current to the far zone field, depends on the source geometry and can be analyzed by its singular value decomposition. Here, first, we present useful upper bounds about the number of degrees of freedom for some 2D source geometries (i.e. for elliptical and parabolic arc sources) and examine the role of two different representation variables. These results were obtained from asymptotic arguments and allow to define the maximum number of independent sources and patterns that can be radiated by each geometry. They are verified to fit the numerically computed ones, too. Next, we examine the point source reconstructions by considering the point spread function. An approximate closed form evaluation reveals that the arc length representation variable leads to a space invariant behavior. The role of the source electrical length in determining the number of degrees of freedom is pointed out, too. Finally, the radiation properties of different source geometries are compared by means of a synthetic index and examples of radiation pattern synthesis and array diagnostics confirm the need to investigate the role of the source geometry.
\end{abstract}

Index Terms - Antenna synthesis, Conformal antennas, Inverse source problem, Number of Degrees of Freedom, Radiation operator, Point Spread Function, Singular Values Decomposition.

\section{INTRODUCTION}

$\mathrm{T}$ HE development of conformal antennas array [1] and the increasing interest in their applications is due to several reasons. First, aero dynamic advantages arise for aircraft (such as UAV [2, 3] and SAR [4] platforms) and vessels, but they could also be exploited for the realization of train antennas, car radio antennas, and cellular base station antennas, also for the demanding 5G networks [5, 6]. Wide-angle and full solid angle scanning capability are often pursued for a variety of applications, such as both ground based [7] and on board [8] surveillance radar. In some advanced application where flexible materials are employed, the shape of an antenna array becomes a new parameter in the synthesis of a shaped beam [9].

Unfortunately, there is a lack of general synthesis methods for conformal antennas [10], that include the synthesis of the source geometry. In fact, all approaches start from a prefixed surface definition. Amongst the most common we might mention: approaches based on constrained least square

Submitted on $15^{\text {th }}$ September 2019.

This work was supported in part by the European Union and the Italian Ministry of University and Research funding under the "Programma Operativo Nazionale Ricerca e Innovazione 2014-2020" and in part by the Italian Ministry of University and Research under the PRIN 2017 Program. approaches [11-13], methods imposing convexity constraints so leading to a convex programming problem $[14,15]$, techniques exploiting linear programming [16], the numerical optimization of non-quadratic cost functional either by vector space projections onto convex set [17] or by stochastic methods $[18,19]$ (sometimes applied to simplified array excitation model [20]), and ad hoc semi-analytical method [21].

While these methods may provide an optimal result for a particular geometry, they cannot allow to perform a general comparison between different source geometries. To this end it may be very useful to devise an approach able to assess in a synthetic way the electrical performances of such different geometries.

Source shapes are compared in [1] by resorting only to geometrical arguments. In particular, efficiency is evaluated as a measure of the antenna surface in the scan direction as the ratio between the projected area along this direction and the actual surface area. In this way no electrical performance is accounted for.

As the supporting surfaces of the array elements are not flat, it is interesting to investigate how the possibility of locating them along a 2D geometry (if a one dimensional array is considered) may provide a wider scope to the antenna designer so to achieve a wider angular coverage, for instance. On the other hand, limitations may arise for well focused patterns because the unalignment of the elements positions provide significant phase shifts of each individual pattern as soon as we move away from the main beam direction. In order to capture the essential features of the problem and illustrate the main results of the solution approach, hereafter we choose to investigate a slightly more general case, i.e. a $2 \mathrm{D}$ continuous scalar source current whose support is a conic curve, and observe the only component of the radiated far field along the whole round observation angle.

The radiation pattern synthesis problem of a conformal source, together with the source diagnostic one, belong to the realm of the inverse source problem and concern the reconstruction of the radiating currents from the knowledge of the far field. Accordingly, it amounts to inverting the integral relationship connecting them.

In particular, the ill-conditioning [22,23] of the relevant integral operator implies that only a finite number of source current functions can be correctly reconstructed in presence of

Giovanni Leone, Fortuna Munno and Rocco Pierri are with the Dipartimento di Ingegneria, Università della Campania Luigi Vanvitelli, Aversa, I-81031, Italy. (e-mail: giovanni.leone@unicampania.it, fortuna.munno@unicampania.it, rocco.pierri@unicampania.it). 
uncertainties on data, otherwise small noise on data can provide a large error on the reconstructed current. This problem may become particularly serious for the diagnostic of the faulty elements of an array, since if their number is too high it may occur that some excitations may not be diagnosed [24]. For this reason it is important to evaluate the Number of Degrees of Freedom (NDF) [25-27] of the source current in connection to its geometry, as the (finite) number (i.e. the dimension of the subspace) of source current functions that can be stably reconstructed [27].

In addition, this number is also related to the achievable resolution [28], i.e. to the capability of the solution algorithm to reconstruct two close point-like sources. Therefore, the Point Spread Function (PSF) properties are fixed by the NDF. It is clear that a detailed knowledge about the NDF and, hence, the achievable resolution is of primary importance in these kinds of problems.

On the other hand, as well known, the physically realizable radiation patterns are related to the source geometry, and can be related to the NDF, too, which, in turn, also define the number of the (i.e. the dimension of the subspace) radiation pattern functions that can be radiated by a finite energy source: the higher the NDF, the larger the subspace that encompasses more far field patterns for a wider range of applications. Accordingly, an appropriate choice between different source geometries in achieving prescribed pattern specifications can be dictated only by the knowledge of the different far fields that each source may radiate.

Therefore, in this paper we aim at discussing the features of the inverse source problem for some conic geometries in order to establish a way to compare their general radiation properties before undertaking the synthesis procedure of a particular antenna with definite specifications.

In [29], for the first time, we proposed to apply the Singular Value Decomposition (SVD) to the analysis of the radiation operator connecting a source with non rectilinear geometry to the far field. The final goal is to provide a general way of comparing their radiation properties in connection with their geometries by examination of the numerical results of the SVD of the relevant operators. However, the main results about the Singular Values' (SVs) behavior and NDF are only numerical and only a qualitative comparison of some different source geometries is performed.

In [30] a circumference conformal geometry is fully examined by the SVD approach, with the effort of providing closed form evaluations and/or estimation of the NDF. First, for different full angle circles, closed form SVDs are recalled or derived and discussed. Next, an arc of circumference is considered and asymptotic arguments are introduced and numerically validated to provide an estimate of both the NDF and the singular function behavior. To this end, the main result is concerned with the role of the source angle, or, rather, its electrical length, in determining the NDF and, thus, the dimension of the subspace of realizable far field patterns. Finally, the role of the NDF is sketched with a simple numerical example of the synthesis of a focusing beam pointing at different directions.
However, while the circular geometry has been already investigated [31], the general 2D case is still an open problem.

The investigation of these features of the inverse source problem may be performed by resorting to the spectral decomposition of the relevant linear operator connecting the source current to the far field, which is introduced in Section II. This is accomplished by examining its SVD, so as to consider, firstly, the SVs behavior, since they provide the NDF of the source in dependence of its geometry. Since also for a general 2D geometry it is difficult to find an analytical and closed form expression of the NDF, the first goal of this work is to provide a useful upper bound for it to compare with the exact numerically computed one. In Section III this goal is accomplished by asymptotic evaluations of the radiation integral in far zone for some 2D source curve shapes observed upon a full circumference angle. Beside an arc of circumference, two other curves are considered: an arc of ellipse and an arc of parabola Next, the role of the curve parametrization, i.e. the variable describing the source geometry, is investigated and the same asymptotic arguments are employed to provide further upper bounds on the NDF for the same curves as above. Again, they are compared with the numerically computed ones. The new theoretical results of the Section are concerned with not only the upper bounds and/or exact values of the NDF, but also with the strict connection of the NDF with the source electrical length, irrespective of its geometry.

Section IV is devoted to the PSF analysis for the two considered parametrization variables in order to confirm and mathematically explain the previous results. In fact, the evaluation of the PSF is commonly employed in the inverse problem literature to provide a measure of the algorithm performances about the resolution capabilities. First a closed form original approximated expression is introduced; its discussion leads to point out the role of the arc length variable, as a spatially invariant behavior is observed; the approximated expression is also compared with the exact one with a good agreement.

Section V aims at applying the previous results to the antenna realm by means of a comparison between four source geometries, with the same length. First the NDF are numerically computed and their dependence on the electrical length is verified. Next, in order to establish the terms of a comparison of their radiated fields, a synthetic index, the Spectral Content Function (SCF), is introduced. Its connection with the pertinent PSF is highlighted and it results as a sort of an average of the PSFs.

The index should help an antenna designer in comparing different geometries according to the required pattern specifications. Accordingly, some numerical examples of pattern synthesis are provided and the behavior of different source geometries are understood in terms of the corresponding SCFs. In addition, an application to conformal array antenna diagnostics, highlights the role of the knowledge of the NDF for reliable results.

Conclusions end the paper in Section VI. 


\section{The Mathematical Model}

In our discussion, we suppose that the source is embedded in a homogeneous medium with dielectric permittivity $\varepsilon_{0}$ and magnetic permeability $\mu_{0}$. In order to simplify the mathematical notation, we assume invariance along the $y$ axis and $y$-directed electric field $E$, so that the problem is scalar. The considered source conic curves are the parabolic and elliptical arcs, over which an angularly variant current density $J$ is supported. For the sake of comparison, results for the rectilinear and circumference arc are reported within the paper, too. The source domain is described by the polar coordinates $r(\phi)$ and $\phi$ : the radial coordinate $r(\phi)$ depends on the considered source geometry, while the angular variable $\phi$ spans between $-\alpha$ and $\alpha$, with $\alpha \in[-\pi / 2, \pi / 2]$. The reference system is centered in the focus $F$ for both the curves (Fig. 1).

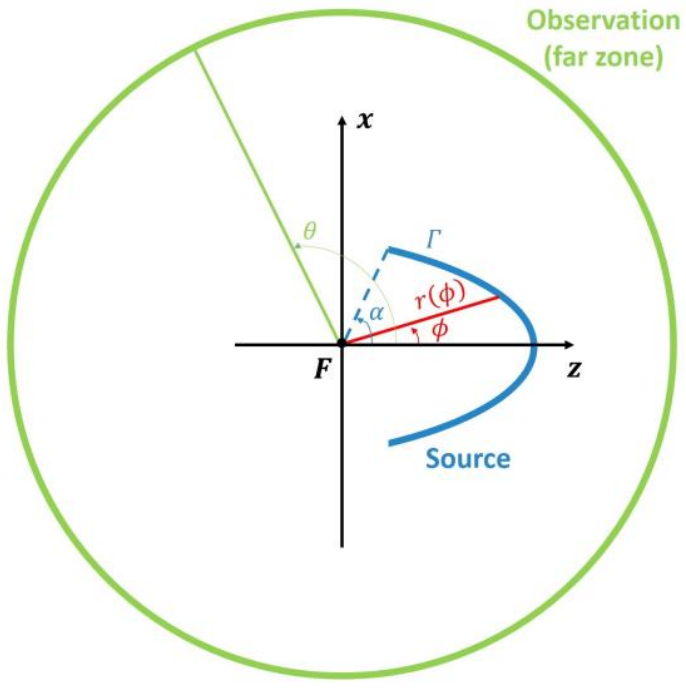

Fig. 1. Relevant to the geometry.

The radiated field $E$ is collected in far zone. When the $E$ function is observed versus the angular variable $\theta$, the radiation operator $\mathcal{A}$ connecting the source current to the far field can written, apart from an inessential factor, as

$E(\theta)=\int_{-\alpha}^{\alpha} J(\phi) e^{j \beta r(\phi) \cos (\theta-\phi)}\left\|\underline{\gamma}^{\prime}(\phi)\right\| d \phi=\mathcal{A}(J)$

where $\beta=2 \pi / \lambda, \quad \lambda \quad$ is the wavelength, $\underline{\gamma}(\phi)=$ $(r(\phi) \sin \phi, r(\phi) \cos \phi)$ is a bijective parametric representation of the curve $\Gamma$ over which the integration is performed and $\left\|\underline{\gamma}^{\prime}(\phi)\right\| d \phi$ is the differential element of $\Gamma$. Equation (1) defines the radiation operator

$\mathcal{A}: J \in L_{[-\alpha, \alpha]}^{2} \rightarrow E \in L_{[-\pi, \pi]}^{2}$

where $J$ and $E$ are assumed to belong to the set of square integrable functions $L_{[\cdot]}^{2}$ supported over the interval specified by the subscript. For a rectilinear $x$ oriented source, when the $\sin \theta$ observation variable is used, (1) becomes the usual Fourier transform relationship between the far zone field and the source current. On the contrary, for a conformal source, the kernel of
(1) is of more general type, it depends on the particular source geometry and deserves a careful investigation.

Since the operator $\mathcal{A}$ is compact, we can compute its Singular Value Decomposition (SVD) [23] made up by the left singular functions $\left\{u_{n}\right\}$, the singular values (SVs) $\left\{\sigma_{n}\right\}$ and the right singular functions $\left\{v_{n}\right\}$. The importance of SVD is twofold: on the one hand it allows us to identify orthonormal bases for the operator range and the operator domain $\left(\left\{v_{n}\right\}\right.$ and $\left\{u_{n}\right\}$, respectively), and on the other hand, it enables to establish the number of significant SVs that may provide the NDF [32], since for an integral operator such as the one in (1) it is well known that the SVs exhibit a step-like behavior. However, both the number of SVs and an analytical SVD are not exactly known for a generic $2 \mathrm{D}$ source geometry, except for the circular source geometry [31]. The lack of knowledge in this area justifies the interest in finding an upper bound to the NDF for such geometries.

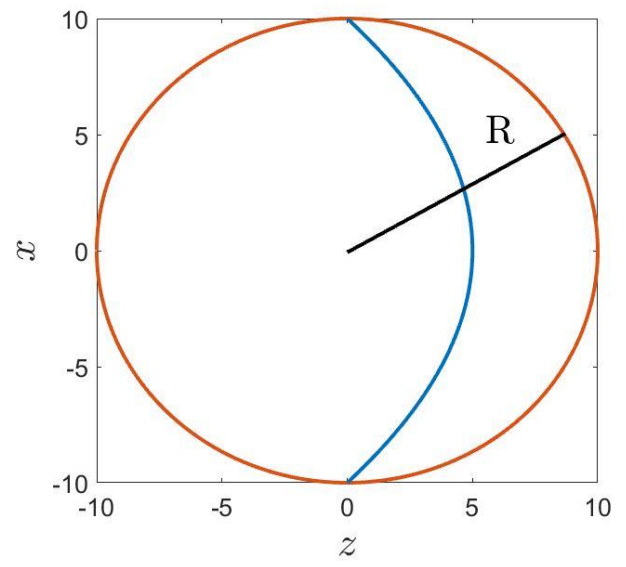

Fig. 2. The smallest circular source (red line) with radius $\mathrm{R}$ including an arc source (blue line).

For the sake of comparison, the smallest circular source including the source is considered (Fig. 2), as exact results about it are the only available ones for this kind of problem. To this end, we recall that for a circular source the maximum number of basis functions representing the source in a stable way can be found exactly and this number provides the NDF. Therefore, upper bounds for the NDF of any conic curve can be provided by considering the smallest circular source circumscribing the source arcs, that is [29],

$$
N_{c}=2[\beta R]+1
$$

where $R$ represents the circle radius and ['] stands for integer part. In the following estimates, which are better than $N_{c}$, are introduced.

\section{NDF ANALYSIS}

The goal of this section is to discuss the NDF of an elliptical and parabolic arc source. This would require to compute the SVs of the relevant operators (1), which, unfortunately, cannot be performed in closed form. Therefore, analytical upper bounds are derived adopting Fourier series expansions for the 
source current functions and following asymptotic reasoning. In addition, the role of the source current variable is examined by assuming, first, that the source geometry is parametrized by the angle coordinate (Subsection A) and, next, by the arc length variable (Subsection B). Finally, the numerical evaluations of the NDF provide support to this analysis (Subsection C).

\section{A. Angular parametrization}

Let us first rewrite (1) as

$E(\theta)=\int_{-\alpha}^{\alpha} J(\phi) e^{j(\beta p)(r(\phi) / p) \cos (\theta-\phi)}\left\|\underline{\gamma}^{\prime}(\phi)\right\| d \phi$

where $p$ is a geometrical parameter much larger than wavelength.

Since the source current right singular functions $\left\{v_{n}\right\}$ are not known analytically, we resort to representing the current function by Fourier harmonics, i.e. as

$J(\phi)=\sum_{m=-\infty}^{\infty} c_{m} \frac{e^{j \frac{m \pi}{\alpha} \phi}}{\sqrt{2 \alpha}}$.

and aim at evaluating its maximum spectral content. Since each current Fourier harmonic contributes to the radiated field as

$\mathcal{A}\left(\frac{e^{j \frac{m \pi}{\alpha} \phi}}{\sqrt{2 \alpha}}\right)=\int_{-\alpha}^{\alpha} \frac{e^{j \frac{m \pi}{\alpha} \phi}}{\sqrt{2 \alpha}} e^{j(\beta p)(r(\phi) / p) \cos (\theta-\phi)}\left\|\underline{\gamma}^{\prime}(\phi)\right\| d \phi$

stationary phase approximation arguments are proposed in order to appreciate how many harmonics $|m|<M$ are required to represent $J$, provided $\beta p \gg 1$.

From the discussion in Appendix A, the maximum number of Fourier harmonics providing a significant contribution to the far field is $N_{e}^{\phi}=2 M+1$ with

$M=\left[\frac{\alpha \beta p}{\pi} \frac{(1+e)}{(1+e \cos \alpha)^{2}}\right]$

Since the Fourier harmonics do not form an "extremal basis", i.e. are not the singular functions of $\mathcal{A}, N_{e}^{\phi}$ provides an upper bound to the NDF.

Finally, we can notice directly from the expression of $N_{e}^{\phi}$ that when $e=0, \alpha=\pi / 2$ and, consequently, $r(\phi)=p=R$ (the case of a semi-circumference source) the value predicted for $N_{e}^{\phi}$ is $\beta R$, in agreement with the results of [31].

When the source is a parabolic arc, the above reasoning can be repeated and the arguments of Appendix A lead to deduce, that the maximum number of Fourier harmonics is $N_{p}^{\phi}=2 M+$ 1 , with

$M=\left[\frac{\alpha \beta p}{2 \pi \cos ^{3}\left(\frac{\alpha}{2}\right)}\right]$

Once again, this number represents an upper bound for the NDF.

\section{B. Arc length parametrization}

Let us investigate a different curve parametrization, that is the arc length $s(\phi)$ variable, defined by

$s(\phi)=s(-\alpha)+\int_{-\alpha}^{\phi}\left\|\underline{\gamma}^{\prime}(t)\right\| d t$

where, if $L$ represents the source total length, $s(-\alpha)$ is set to $-L / 2$. Operator (4) is now rewritten as

$E(\theta)=\int_{-L / 2}^{L / 2} J(s) e^{j(\beta p)(r(\phi(s)) / p) \cos (\theta-\phi(s))} d s=\widehat{\mathcal{A}}(J)$

and the previous asymptotic approach can be followed for $\beta p \gg 1$ so to lead to upper bounds for the NDF of (10).

This time, the source current function is represented as a Fourier harmonics superposition in the $s$ variable, that is,

$J(s)=\sum_{m=-\infty}^{\infty} c_{m} \frac{e^{j \frac{m 2 \pi}{L}} s}{\sqrt{L}}$

and, again, we aim at evaluating the contribution of each current harmonic to the far field.

The maximum allowable harmonic order is now given by

$M=\left[\frac{L}{\lambda} v\right]$

where $v=(1+e) / \sqrt{1+e^{2}+2 e \cos \alpha}$. In other words, $M$ is proportional to the electrical length of the source by a coefficient $v$ which is always greater than one. Accordingly, the maximum number of Fourier harmonics needed to represent the source current is $N_{e}^{S}=2 M+1$, so providing another upper bound for the NDF.

Turning the attention to the parabolic arc and repeating the same steps (see Appendix A) as above we find out that, the maximum Fourier harmonic order $m$ allowing for a real solution of the stationary phase condition is provided by

$M=\left[\frac{L}{\lambda}\right]$

and, consequently, the maximum number of Fourier harmonics is $N_{p}^{s}=2 M+1$.

In this way, we can point out that, again, a link between the NDF asymptotic upper bound and the length source is found, as in (12).

\section{Exact NDF}

Here, the effectiveness of the four upper bounds derived in the previous subsections is tested by means of the numerical computation of the SVs. This is performed from the SVD of (4) and (10) with a sufficiently dense discretization of both the source and observation spaces. Then the actual NDF, denoted by $N_{a c t}^{\phi}$ and $N_{a c t}^{s}$ respectively, corresponds approximatively to the index where the knee of the SVs curve occurs, i.e. when the exponential decay of the SVs starts.

In Fig. 3, once $p$ is fixed, the exact, numerically computed, SVs behavior of both operators $\mathcal{A}$ and $\widehat{\mathcal{A}}$ is shown for the 
elliptic arc, for different $\varepsilon$ and compared with that $N_{e}^{\phi}$ and $N_{e}^{s}$. It results that, when $\alpha$ is not large, the above deduced upper bounds provide a very good estimation of the actual NDF.
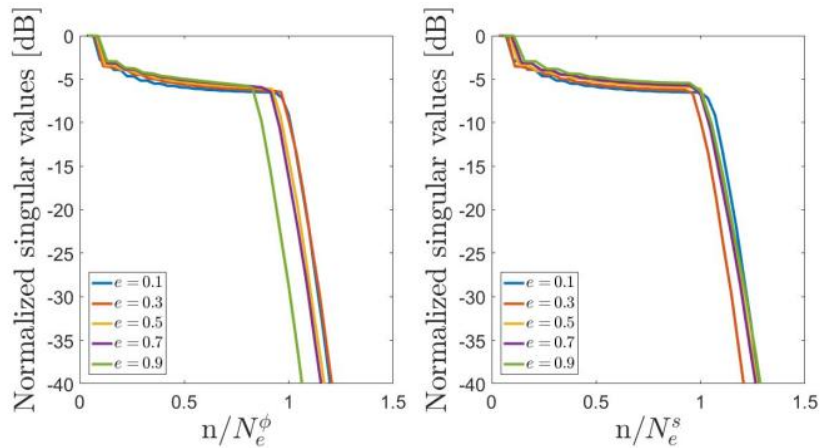

Fig. 3. Behavior of the exact SVs of the operators $\mathcal{A}$ (left panel) and $\hat{\mathcal{A}}$ (right panel) for an elliptical arc with $p=10 \lambda$ and $\alpha=\pi / 4$, by varying the eccentricity $e$.

In Fig. 4 the exact, numerically computed, SVs behavior of both operators $\mathcal{A}$ and $\widehat{\mathcal{A}}$ is shown for the parabolic arc, for different $p$ and compared with that $N_{p}^{\phi}$ and $N_{p}^{s}$. In particular, $N_{p}^{S}$ is always very close to $N_{a c t}^{s}$ and that that the estimation $N_{p}^{\phi}$ is closer to $N_{\text {act }}^{\phi}$ when $\alpha$ is not large. Therefore, it can be concluded that in all circumstances the asymptotic analysis can be useful in defining the dimension of both the current and the field representation subspaces.

These conclusions are further supported by the quantitative sample results reported in Tables I-III. Here, we include a further comparison with the loose upper bounds $N_{c}$ (i.e. (3) of Section II), obtained by considering the smallest circle including the source arc. This result is the only available general upper bound involving a non rectilinear source geometry.

For the elliptic arc, the smallest including circle has a radius

$R=\frac{p \sin \alpha}{1+e \cos \alpha}$

to be used in (3). It can be observed that $N_{c}$ is always larger than $N_{e}^{\phi, s}$ (see Tables I and II),

For the parabolic arc, the smallest including circle has a radius

$R=\frac{p \sin \alpha}{1+\cos \alpha}$

It can be observed in Table III that $N_{p}^{\phi, s}$ is always smaller than $N_{c}$ for this curve, too.

Results of Tables I-III provide another two very interesting conclusions.

First of all, $N_{\text {act }}^{\phi}$ and $N_{\text {act }}^{S}$ are nearly equal in all circumstances. This means that the NDF does not depend on the curve parametrization but only on its geometry. The curve parametrization affects only the behavior of the SVs before the knee, but does not change its occurrence.

On the other hand, $N_{p}^{s}$ is nearly twice the curve electrical length (see (13)), while $N_{e}^{s}$ is very close to it (see (12)), and both approximate $N_{\text {act }}^{s}$ very well.
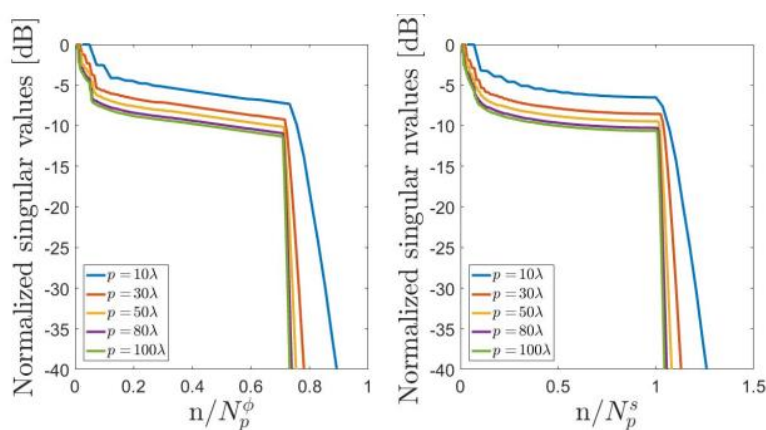

Fig. 4. Behavior of the exact SVs of the operators $\mathcal{A}$ (left panel) and $\hat{\mathcal{A}}$ (right panel) for a parabolic arc with $\alpha=3 \pi / 8$, by varying the semi-latus rectum.

Therefore, it is apparent that the electrical length of the source, rather than its geometry, plays an important role in the evaluation of the actual NDF. As a numerical confirmation of this result, we consider three conic sources with the same length $L$, i.e. semi-circumference, parabolic and elliptical arcs. An extensive numerical analysis has been performed and a typical result of the SVs of both operators $\mathcal{A}$ and $\widehat{\mathcal{A}}$, for $L=20 \lambda$ is reported in Fig. 5. In particular the exponential decay of the SVs starts at an index approximately equal to $N_{a c t}^{\phi}=N_{a c t}^{S}=40$ for every curve, irrespective of the geometry parametrization.
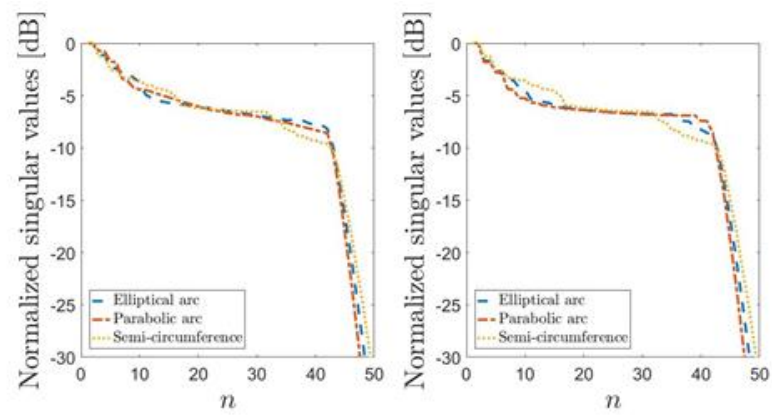

Fig. 5. Behavior of the exact SVs for elliptical, parabolic and circumference arcs with the same $20 \lambda$ length and $\alpha=\pi / 2$. The left panel refers to the operator $\mathcal{A}$ while the right panel refers to the operator $\hat{\mathcal{A}}$.

Accordingly, both by the asymptotic upper bounds and by the numerical results, as a second outcome of the Section, the relation linking the NDF with the source length is

$N_{\text {act }}^{s} \approx\left[\frac{2 L}{\lambda}\right]$

where $\lambda$ is the wavelength. In next Section a further explanation for this result is provided.

\section{PSF ANALYSIS}

Within an inverse source problem, it is worth appreciating the capabilities of the solution algorithm in reconstructing a source current in a stable way. A typical performance index is connected to the possibility of distinguishing between two close point sources, which can be useful also for an array antenna source when its diagnostics is of interest, for the identification 
of faulty elements, for instance. In this circumstance, the PSF is to be considered, which is defined as the reconstruction of a point-like source by the inversion scheme, and provides a measure of the resolution in the reconstruction by its main lobe width [33]. In addition, the behavior of its main lobe can provide further interesting information, since similar main lobes centered at different source points would imply the same reconstruction along the whole source domain, i.e. a space invariant result; on the contrary, if the PSF is space variant, different resolution can be expected at different source points.

Therefore, hereafter, first we evaluate the PSFs associated to the relevant operators (1) and (10). If we start by considering the $\phi$ variable and the $\mathcal{A}$ operator, by its definition, the PSF can be expressed as the impulsive response to the system made up by the cascade of the direct operator and its regularized inverse, i.e.

$\operatorname{PSF}\left(\phi, \phi_{0}\right)=\mathcal{A}^{-1} \mathcal{A}\left(\delta\left(\phi^{\prime}-\phi_{0}\right)\right)$

where $\delta(\cdot)$ represents a Dirac impulsive function centered at the source domain point $\phi_{0}$, supported over the source domain, and providing the point-like source location. Accordingly, when a truncated SVD (TSVD) inversion scheme is exploited, the PSF results as

$\operatorname{PSF}\left(\phi, \phi_{0}\right)=\sum_{i=1}^{N D F} v_{i}(\phi) v_{i}^{*}\left(\phi_{0}\right)$

and its evaluation requires the knowledge of the right singular functions $\left\{v_{n}\right\}$, which, in general, can be gained only by the numerical computation of the SVD of the operator.

However, in order to overcome this difficulty, we are going to introduce an approximated evaluation of the PSF. In fact, since the SVs exhibit a step-like trend, we can resort to the adjoint operator to perform the (regularized) inversion [34], keeping in mind that we will obtain an approximated result because the actual SVs are not constant.

So we introduce the approximate PSF as:

$\widetilde{P S F}\left(\phi, \phi_{0}\right)=\mathcal{A}^{\dagger} \mathcal{A}\left(\delta\left(\phi^{\prime}-\phi_{0}\right)\right)$

and it turns out that

$\widetilde{P S F}\left(\phi, \phi_{0}\right)=\sum_{i=1}^{N D F} \sigma_{i}^{2} v_{i}(\phi) v_{i}^{*}\left(\phi_{0}\right)$

By definition, the adjoint operator of $\mathcal{A}, \mathcal{A}^{\dagger}$, satisfies the relationship

$<\mathcal{A}(J), E>_{L_{[-\pi, \pi]}^{2}}=<J, \mathcal{A}^{\dagger}(E)>_{L_{[-\alpha, \alpha]}^{2}}$

where $\langle\cdot, \cdot\rangle$ stands for the scalar product within the appropriate space. Accordingly, it can be written as

$\mathcal{A}^{\dagger}(E(\theta))=\left\|\underline{\gamma}^{\prime}(\phi)\right\| \int_{-\pi}^{\pi} E(\theta) e^{-j \beta r(\phi) \cos (\theta-\phi)} d \theta$

By inserting (1) into (24) and resorting to the sifting property of the impulse, (21) becomes

$\widetilde{P S F}\left(\phi, \phi_{0}\right)=\left\|\underline{\gamma}^{\prime}(\phi)\right\|\left\|\underline{\gamma}^{\prime}\left(\phi_{0}\right)\right\| \cdot$
$\int_{-\pi}^{\pi} e^{j \beta\left[\left(x\left(\phi_{0}\right)-x(\phi)\right) \sin \theta+\left(z\left(\phi_{0}\right)-z(\phi)\right) \cos \theta\right]} d \theta$

with $x(\phi)=r(\phi) \sin \phi$ and $z(\phi)=r(\phi) \cos \phi$. Let us, now, focus on the integral in (25). First, it is useful to recall the integral expression of the Bessel function of zero-th order as

$J_{0}(\gamma)=\frac{1}{2 \pi} \int_{-\pi}^{\pi} e^{j \gamma \cos (\theta-\nu)} d \theta$

for arbitrary $v$. Next, we denote with $\underline{\rho}=(x, z)$ and $\underline{\rho}_{0}=$ $\left(x_{0}, z_{0}\right)$ two points of the $x-z$ plane. Finally, the phase function in (25) is cast under a cosine function as in (26) so that the integral in (25) is exactly retrieved as

$\int_{-\pi}^{\pi} e^{j \beta\left[\left(x-x_{0}\right) \sin \theta+\left(z_{0}-z\right) \cos \theta\right]} d \theta=$
$2 \pi J_{0}\left(\beta \sqrt{\left(x\left(\phi_{0}\right)-x(\phi)\right)^{2}+\left(z\left(\phi_{0}\right)-z(\phi)\right)^{2}}\right)$

Therefore, the approximated PSF can be expressed as

$\widetilde{P S F}\left(\phi, \phi_{0}\right)=2 \pi\left\|\underline{\gamma}^{\prime}(\phi)\right\|\left\|\underline{\gamma}^{\prime}\left(\phi_{0}\right)\right\| J_{0}\left(\beta\left|\underline{\rho}_{0}-\underline{\rho}\right|\right)$

From (28) it is straightforward to appreciate that, strictly speaking, the approximated PSF is dependent on the point source position and, hence, exhibits spatial variance. However, the outer functions depending on the curve parametrization, are smooth functions of the independent variable, so their contribution to the spatial variance can be neglected and discussion can focus only on the Bessel function factor, which represents a space invariant function.

On the other hand, when we consider the $\widehat{\mathcal{A}}$ operator and repeat the same discussion for the corresponding approximate PSF function, it results

$\widetilde{P S F}\left(s, s_{0}\right)=$ $\int_{-\pi}^{\pi} e^{j \beta\left[r\left(\phi\left(s_{0}\right)\right) \cos \left(\theta-\phi\left(s_{0}\right)\right)-r(\phi(s)) \cos (\theta-\phi(s))\right]} d \theta=$

$2 \pi J_{0}\left(\beta\left|\underline{\rho_{0}}-\underline{\rho}\right|\right)$

where $\underline{\rho}=(x(s), z(s))$ and $\underline{\rho}_{0}=\left(x\left(s_{0}\right), z\left(s_{0}\right)\right)$ denote the observation point and the impulsive source location, respectively. Then the PSF is a space invariant function.

Let us now consider the first lobe of the Bessel function in (28) and (29), whose argument is the distance between the observation point and the impulsive source location. For observation points close to the source location and for small curvature, this distance can be well approximated by the difference in the corresponding arc lengths, just like an arc can be approximated by its secant. Then, $\left|\underline{\rho}_{0}-\underline{\rho}\right| \simeq\left|s-s_{0}\right|$, and within its main lobe, the approximated PSF is provided by

$\widetilde{P S F}\left(s, s_{0}\right) \simeq 2 \pi J_{0}\left(\beta\left|s-s_{0}\right|\right)$. 
This result implies that, when the arc length observation variable is adopted, an approximately space invariant PSF is obtained.

A comparison between the analytical result in (28) and the numerically computed exact PSFs is presented in the upper panels of Figs 6 and 7, while in the lower panels the PSF for the $\widehat{\mathcal{A}}$ operator is approximated by (30). We can notice that the PSFs main lobe width is well predicted in every case.
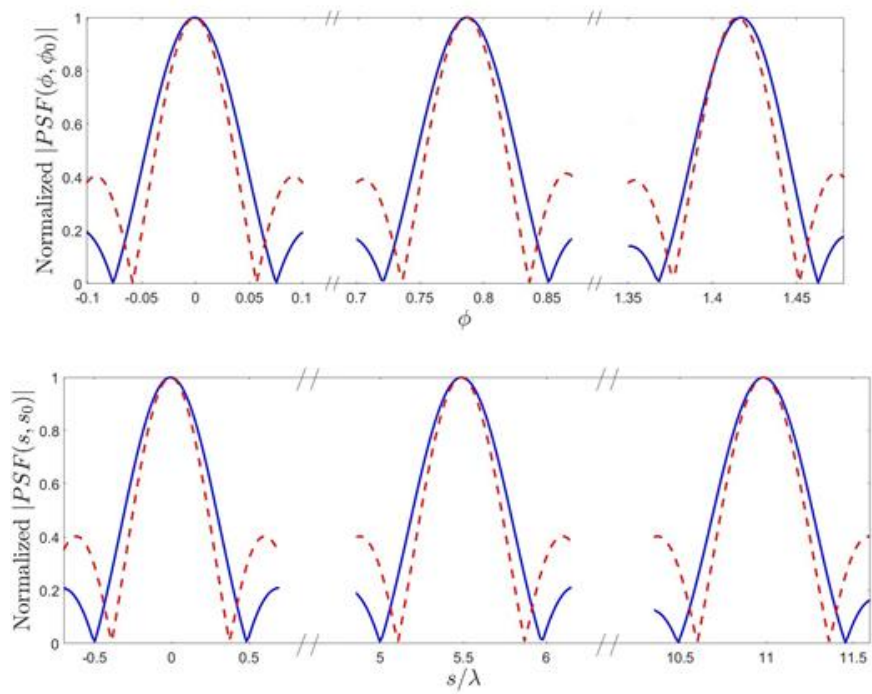

Fig. 6. Normalized PSFs main lobe of operator $\mathcal{A}$ (upper panel) and $\hat{\mathcal{A}}$ (lower panel) for an elliptic arc with $p=10 \lambda, \alpha=\pi / 2$ and $e=0.5$. The blue curves represent the numerically computed PSF, while the red curves are the approximated ones. The source points are located at $\phi_{0}=0,0.79,1.41$ for operator $\mathcal{A}$ and at the corresponding arc lengths $s_{0}$ for the $\hat{\mathcal{A}}$ operator.
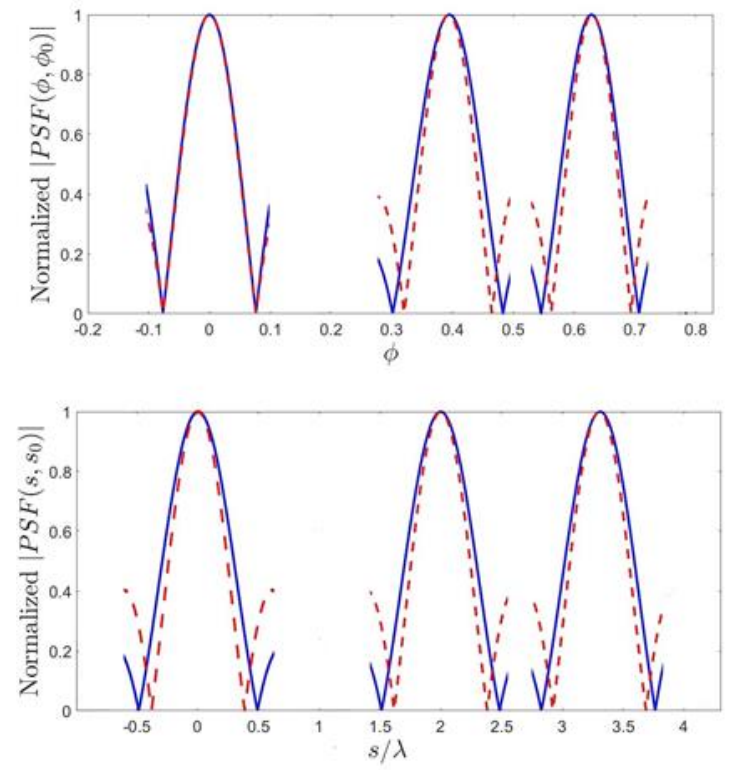

Fig. 7. Normalized PSFs main lobe of operator $\mathcal{A}$ (upper panel) and $\hat{\mathcal{A}}$ (lower panel) for a parabolic arc with $p=10 \lambda$ and $\alpha=\pi / 4$. The blue curves represent the numerically computed PSF, while the red curves are the approximated ones. The source points are located at $\phi_{0}=0,0.39,0.63$ for operator $\mathcal{A}$ and at the corresponding arc lengths $s_{0}$ for the $\hat{\mathcal{A}}$ operator.

From the analytical (although approximated) expression of the PSF for the arc length variable, a way to estimate the NDF can be derived as the number of point sources that can be resolved by the regularized inversion scheme. Two points like sources are reconstructed when their distance is larger than the width of the main lobe of the PSF, which can be assumed as the interval where the modulus of the PSF is half than its maximum [33]. By (30) the width of the PSFs main lobe at half height is $\Delta s \approx 0.484 \lambda$ and it is constant along the domain, because of the found space invariance. Accordingly, we can count the number of the PSFs main lobes covering the source domain and overlapping at their half heights. Hence, the NDF can be computed approximately as $[L / \Delta s]$. For example, for an elliptic arc with $p=10 \lambda, \varepsilon=0.5$ and $\alpha=\pi / 2$, the source length is $L \approx 25.3 \lambda$ returning an NDF evaluation approximately equal to 52 , that is very close the actual one (see Table I). Analogously, if we consider a parabolic arc with $p=10 \lambda$ and $\alpha=\pi / 4$, the source length will be $L \approx 8.52 \lambda$ leading to an estimation of the NDF of about 17, that is exactly the actual value (see Table III).

Therefore, this analysis confirms that the actual NDF is connected to the curve electrical length, instead of its shape, as also found in the previous Section.

\section{ANTENNA APPLICATIONS}

In order to point out the role of the geometry in the capability of a source to radiate a desired field, hereafter we compare different source geometries fixing their electrical length and their angular extension to a common value. The first constraint is derived from the observation of the previous Sections about its important role in defining the NDF. Therefore, the dimension of the subspaces spanning the radiation patterns of the various sources are the same, while the actual set of realizable patterns, as defined by the left singular functions $\left\{u_{n}\right\}$ associated to the relevant geometry, may differ.

The PSF turns out to be also a good figure of merit for the radiation properties of a source when it is referred to the observation domain. In this case, the PSF in $\theta$, named $P S F_{F F}\left(\theta, \theta_{0}\right)$, describes the capability of a source to radiate a far field focusing in a given direction $\theta_{0}$. The $P S F_{F F}$ is defined as the impulsive response to the system consisting of the cascade of the regularized inverse and the direct operator and is given by

$\operatorname{PSF}_{F F}\left(\theta, \theta_{0}\right)=\mathcal{A}_{\mathcal{A}} \mathcal{A}^{-1}\left(\delta\left(\theta^{\prime}-\theta_{0}\right)\right)$

When a truncated SVD (TSVD) inversion scheme is adopted, the $P S F_{F F}$ writes explicitly as

$P S F_{F F}\left(\theta, \theta_{0}\right)=\sum_{i=1}^{N D F} u_{i}(\theta) u_{i}^{*}\left(\theta_{0}\right)$

An interesting source property for omni-directional coverage applications might be provided, for example, by a spaceinvariant $P S F_{F F}$, meaning that the source is able to radiate the same beam in different directions. However, to appreciate this feature it would be necessary to compute (32) by varying $\theta_{0}$ explicitly. A more synthetic way to identify the radiation properties of a source can be achieved by resorting to the 
Spectral Content Function (SCF) defined as

$\operatorname{SCF}(\theta)=\sum_{i=1}^{N D F}\left|u_{i}(\theta)\right|^{2}$

since it can be shown to be related to $\operatorname{PSF}_{F F}\left(\theta, \theta_{0}\right)$.

The $\operatorname{SCF}(\theta)$, indeed, may describe the average behavior of the PSF squared magnitude along the observation domain, as follows. If we integrate the squared magnitude of the $P S F_{F F}$ with respect to $\theta$ along the whole observation interval, we obtain

$\int_{-\pi}^{\pi}\left|P S F_{F F}\left(\theta, \theta_{0}\right)\right|^{2} d \theta=$

$\int_{-\pi}^{\pi} \sum_{i=1}^{N D F} u_{i}(\theta) u_{i}^{*}\left(\theta_{0}\right) \sum_{j=1}^{N D F} u_{j}^{*}(\theta) u_{j}\left(\theta_{0}\right) d \theta$

By interchanging the summation and the integral, (34) becomes

$\sum_{i=1}^{N D F} \sum_{j=1}^{N D F} u_{j}\left(\theta_{0}\right) u_{i}^{*}\left(\theta_{0}\right) \int_{-\pi}^{\pi} u_{j}^{*}(\theta) u_{i}(\theta) d \theta$

and, thanks to the orthonormality of the left singular functions, we finally obtain

$\int_{-\pi}^{\pi}\left|P S F_{F F}\left(\theta, \theta_{0}\right)\right|^{2} d \theta=\sum_{i=1}^{N D F}\left|u_{i}\left(\theta_{0}\right)\right|^{2}=\operatorname{SCF}\left(\theta_{0}\right)$

that is exactly the SCF defined in (33).

Indeed, if the $P S F_{F F}$ is space-invariant, namely, it does not depend on the particular observation point $\theta_{0}$ where the impulsive source is applied, $\operatorname{PSF}_{F F}\left(\theta, \theta_{0}\right)=P S F_{F F}\left(\theta-\theta_{0}\right)$. Then

$\int_{-\pi}^{\pi}\left|P S F_{F F}\left(\theta-\theta_{0}\right)\right|^{2} d \theta_{0}=S C F(\theta)=$ constant

is no longer a function of $\theta_{0}$ but only of the observation variable $\theta$. Function (33) can be also read as the contribution of all significant singular functions to every direction of the observation domain; hence, we can expect that the directions where it is low can provide the angular domain where the far field patterns are generally not well approximated worse.

In order to perform a comparison for different source geometries, first, we plot the SCF and, next, we provide some examples of far field pattern synthesis. Since the electrical length of the sources provides a very good approximation to the NDF, we assume that all sources have the same length. In addition to the elliptical and parabolic arcs, we also consider a line and a semi-circumference source, since they may provide a source geometry with better unidirectional behavior and a "more omnidirectional" one, respectively (fig. 8). The sources' length $L$ chosen for the examples is $20 \lambda$, while the conic sources angle $\alpha$ is set to $\pi / 2$.

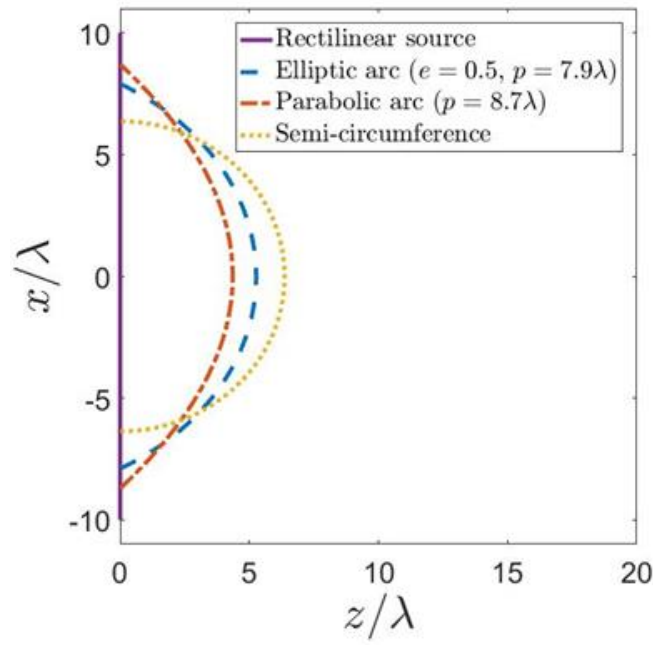

Fig. 8. The source geometries of the examples of Section V.

In Fig. 9 the behavior $S C F(\theta)$ is depicted for all the four geometries and, since $\operatorname{SCF}(\pi-\theta)=\operatorname{SCF}(\theta)$, is plotted only on half observation domain.

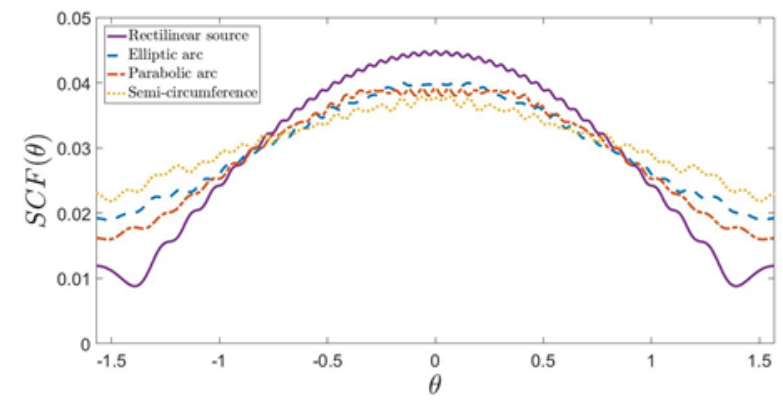

Fig. 9. Behavior of the $\operatorname{SCF}(\theta)$ on half domain $(\theta \in[-\pi / 2, \pi / 2])$ for different curves, when $L=20 \lambda$ and, for the conic sources, $\alpha=\pi / 2$.

From the behavior of these functions we can expect that around $\theta=0$, where all the curves are rather flat, all these geometries can radiate similar patterns, especially the rectilinear and the parabolic ones exhibiting the flattest behavior. On the contrary, for observation directions $|\theta| \rightarrow$ $\pi / 2$, where markedly different behaviors are observed with different decay rates, the semi-circumference source may provide focusing patterns similar to ones radiated in other directions. A beam radiated by a rectilinear source, pointing at larger observation directions, may not be well approximated. An elliptic source and a parabolic one may radiate patterns with intermediate accuracy.

In order to verify these expectations, we now consider the synthesis of two patterns derived from illustrative antenna applications. The field synthesis procedure proceeds as follows. For an assigned complex far field function $E(\theta)$, the physically realizable radiation pattern $\tilde{E}(\theta)$ is represented by the left singular functions $u_{n}$ associated to each source, as

$\tilde{E}(\theta)=\sum_{n=1}^{N D}\left\langle E(\theta), u_{n}(\theta)\right\rangle u_{n}(\theta)$

i.e. the projection of the $E(\theta)$ function onto the subspace of the 
first pertinent left singular functions.

\section{A. Cosecant Squared Pattern}

The first case concerns the approximation of the cosecant far field pattern

$E(\theta)=\left\{\begin{array}{cc}\csc (\pi / 2-\theta), & \text { for } \theta \in[\pi / 6, \pi / 2-0.15] \\ \csc (0.15), & \text { for } \theta \in(\pi / 2-0.15, \pi / 2-0.1] \\ 0, & \text { elsewhere }\end{array}\right.$

The $\theta$ interval choice meets the need to radiate a cosecant squared pattern with a maximum value reached for $\theta=\pi / 2-$ 0.15 and kept constant until $\theta=\pi / 2-0.1$. This angular interval corresponds to the directions close to the endfire direction with respect the $\mathrm{x}$ axis. The projected fields are shown in Fig. 10, together with the corresponding current function under Fig. 11.

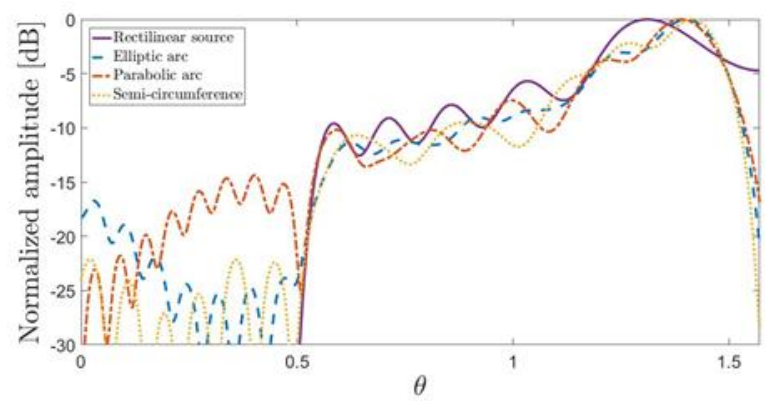

Fig. 10. Normalized $\tilde{E}(\theta)$ fields for the rectilinear, elliptical, parabolic and semi-circumference sources.
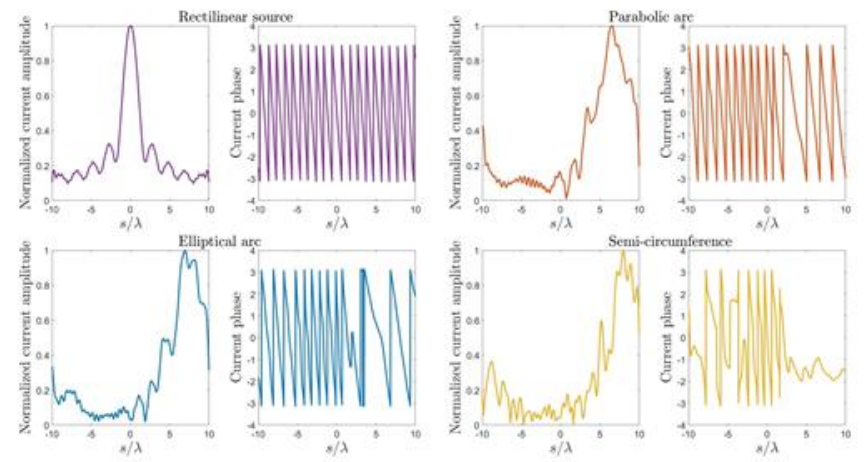

Fig. 11. Excitation currents (amplitude and phase) for the cosecant squared pattern.

In Table IV, the direction of the maximum of the projected field is reported, while in Table $\mathrm{V}$ the ratio between the maximum amplitude of the projected field and its value at the horizon $(\theta=\pi / 2)$ is presented. This analysis is of relevance since in the air traffic control applications, the antenna is required to radiate as little as possible in proximity of the horizon, while, at the same time, the actual peak direction $\tilde{\theta}_{\max }$ of the field should point as close as possible to it.

Since the maximum occurs outside the interval $[-\pi / 4, \pi / 4]$, where all SCFs of fig. 9 exhibit a rather similar behavior, we observe, as expected, that the semi-circumference, followed by the elliptical arc, can guarantee a smaller angular error for the maximum, while, moving from an elliptical arc to a parabolic one and to a rectilinear one, performances degrade. In addition, except for the rectilinear source whose performance in the coverage sector is unacceptable, the side lobe level is lower for the semi-circumference source.

\section{B. Focusing Pattern}

Let us, now, consider a far field function $E(\theta)$ focusing at direction $\theta_{0}$, computed as shown in Appendix B with the goal of providing rather identical beams pointing at different maximum directions for a semi circumference source. It is useful for designing air-surveillance radars with a large angular coverage [35]. Again the synthesis procedure consists in the projection of the $E\left(\theta-\theta_{0}\right)$ function for different $\theta_{0}$ onto the subset of the significant singular functions characterizing each geometry. Fig. 12 provides the application results of (38).
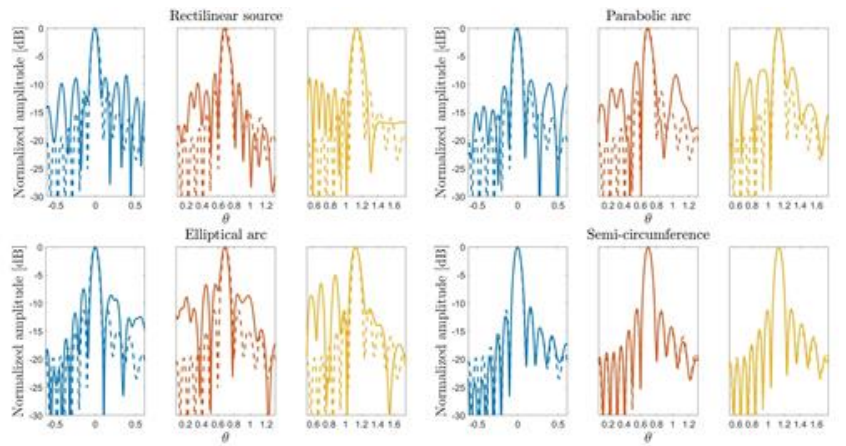

Fig. 12. Normalized $\tilde{E}(\theta)$ (solid lines) and $E(\theta)$ (dashed lines) fields for the rectilinear, elliptical, parabolic and semi-circumference sources focusing forward the directions $\theta_{0}=0$ (blue lines), $\theta_{0}=0.7$ (red lines) and $\theta_{0}=1.13$ (yellow lines).

As can be appreciated and in accordance with the expectations of Fig. 9, the rectilinear source provides the worst results in terms of uniformity of the beam, while the semicircumference source behaves better.

A further quantitative assessment of how well the field radiated from the source follows the desired pattern can be computed resorting to the directivity, defined as

$D\left(\theta_{0}\right)=\frac{\left|\tilde{E}\left(\theta_{0}\right)\right|^{2}}{\frac{1}{2 \pi} \int_{-\pi}^{\pi}|\tilde{E}(\theta)|^{2} d \theta}$

Accordingly, we compare the capability of the different sources of radiating an identical beam pointing at different directions by appreciating to what extent the directivity remains constant.

Table VI shows the values of this parameter for some $\theta_{0}$. As expected, the rectilinear source provides the highest variability of the directivity. In accordance with Fig. 9, the semicircumference performs better, i.e. it provides a uniform pattern and uniform directivity, followed by the elliptical arc.

Some comments about the role of the aspect ratio of the curve geometries, i.e. the ratio between the longitudinal and transverse dimensions, concerning the above results are now in order.

On the whole it can be concluded that a rectilinear source, 
whose aspect ratio is zero, is capable of radiating a well-focused beam along the broadside direction and the width of the main lobe reduces as its electrical length increases, i.e. its transverse dimension. On the contrary, away from that direction the main beam deteriorates and enlarges. This corresponds to the tapered behavior of the $\operatorname{SCF}(\theta)$, which is connected to the angularly variant behavior of the $P S F_{F F}$ in the observation domain.

At the opposite side, a semi-circumference source, whose aspect ratio is 0.5 (as the longitudinal dimension is half than the transverse one), is capable of radiating the same beam pointing at any direction. This corresponds to a constant behavior of the $\operatorname{SCF}(\theta)$, which is connected to the angularly invariant behavior of the $P S F_{F F}$ in the observation domain.

When we move from a rectilinear source to other conic geometries, the extension of the sources along the longitudinal direction is increased with respect to the transverse one. This affects the $\operatorname{SCF}(\theta)$ behavior and makes it flatter. Therefore, conic sources may radiate more similar beams along directions away from broadside, since the longitudinal component of the source geometries provide those contributions to the radiated field that allow to increase focalization. Thus, fixed the transverse extension of the source, the larger the longitudinal extension the more uniform the radiated beams. In fact, we expect that a source with a larger transverse extension is able to radiate a narrower beam toward $\theta_{0}=0$, while a source with a larger longitudinal extension can radiate a narrower beam around $\theta_{0}=\pi / 2$.

\section{Array antenna diagnostics.}

The final numerical example concerns a conformal array antenna, as a special case of a continuous source function. In this case the role of the knowledge of the NDF of the source is emphasized by a diagnostic example, where faulty elements are sought. The crucial point concerns the reliability of inversion algorithm, since the antenna testing engineer must be confident that every set of the excitation coefficients of the array must be recoverable. Due to the role of the NDF in the inversion of (1), a bound on the maximum number $\mathrm{N}_{\text {array }}$ of array elements can be provided. In fact, it can be expected that when $\mathrm{N}_{\text {array }}<\mathrm{NDF}$ the diagnostics procedure will be always reliable. On the contrary, when $\mathrm{N}_{\text {array }}>\mathrm{NDF}$, there may occur sets of excitation coefficients that cannot be reconstructed. In order to prove this point, we consider two conformal equispaced arrays over a $20 \lambda$ long parabolic arc, with $\mathrm{N}_{\text {array }}=40$ and 50 , respectively. The excitation coefficients are arranged so that both arrays radiate a beam focused at $\theta_{0}=0$, but a few (four) faulty elements exist. Figs. 13 and 14 show the reconstructed current functions by TSVD inversion from far zone data. Since NDF=40, as expected, the diagnostics procedure is reliable for the first array, as the presence of faulty elements can be unambiguously detected, while not every set of the excitation coefficients of the second array can be reconstructed, where faulty and active elements are reconstructed with similar accuracy.

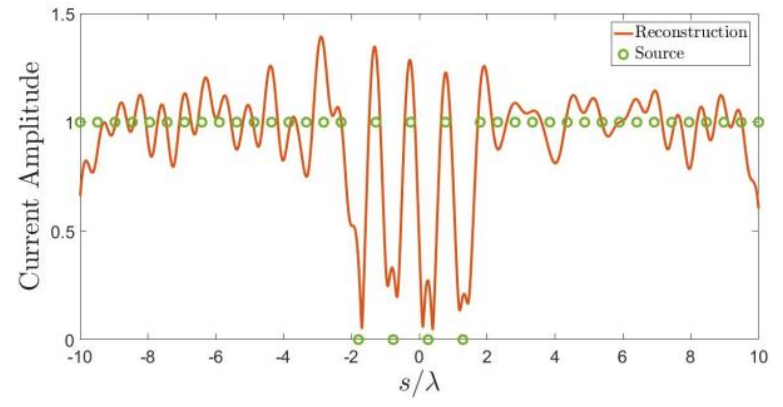

Fig. 13. TSVD reconstruction (red line) of the amplitude of the current function $\mathrm{J}(\mathrm{s} / \lambda)$ for a $20 \lambda$.long parabolic array of 40 elements vs the amplitude of the excitation coefficients of the array elements (green points).

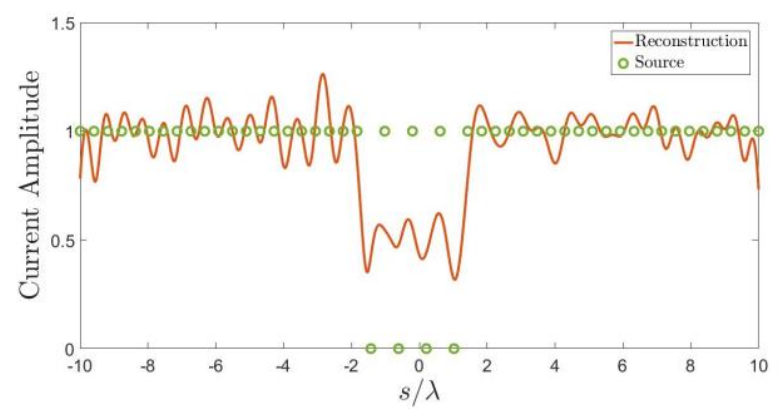

Fig. 14. TSVD reconstruction (red line) of the amplitude of the current function $\mathrm{J}(\mathrm{s} / \lambda)$ for a $20 \lambda$.long parabolic array of 50 elements vs the amplitude of the excitation coefficients of the array elements (green points).

\section{CONCLUSION}

The radiation of conformal, conic 2D sources is investigated via their SVD with the goal to establish a strategy to compare them in a synthetic way, instead of providing a new radiation pattern synthesis scheme. Three points have been raised and analyzed.

First, the investigation of the NDF, which defines the dimension of the subspaces of the source current that can be reliably reconstructed and of the corresponding radiation patterns, is performed by asymptotic reasoning allowing to achieve upper bounds for each considered curve (i.e. an arc of ellipse and an arc of parabola). The role of the parametrization of the source geometry is appreciated, too, so that the arc length variable provides an upper bound of the NDF very close to its actual number. In particular, it is observed that the latter is related only to the electrical length of the curve and not to its shape.

Next, the reconstruction capability of an SVD based inversion scheme is examined via the PSF. By resorting to an approximate evaluation, an analytical expression of the PSF leads to a space invariant behavior which is appreciated for the arc length variable parametrization to span the source domain. This allows to provide an estimate of the NDF by the analysis of the main lobe of the PSF which is related only to the electrical length of the curve and not to its shape, again.

Finally, examples of far field synthesis are reported in order to appreciate the role of the source geometry on the subspace of radiated fields. To this end we have investigated the radiation properties of the conic source geometries in comparison with 
more common ones, like the line and the semi-circumference source. In order to provide a concise function summarizing the radiation behavior in far zone of the considered sources, we have introduced the "spectral content" function and shown that it is related to the PSF referred to the observation domain.

The index should help an antenna designer to compare different geometries according to the required pattern specifications. So a design procedure might proceed as follows. First the electrical length of the source should be fixed according to the expected complexity of the source (for an array source, this entails fixing the number of elements according to feeding requirements as the NDF multiplied by the half wavelength spacing). Next, the source geometry is chosen with the help of the SCF according to the pattern specifications. For instance, if it is required to radiate mainly at broadside directions, all geometries can be expected to behave similarly except the rectilinear one, which exhibits better performances. On the contrary, for near endfire radiation, the semi circumference solution behaves better, although by a bulkier geometry with a large aspect ratio. Then other choices can be compared as well.

The role of the NDF knowledge is emphasized by the application to an example of array diagnostics, too.

The whole analysis clarifies many issues concerning the inverse source problem for non rectilinear sources, with relevant applications in array diagnostics and radiation pattern synthesis of conformal antennas. The extension of the approach to the case of $3 \mathrm{D}$ source geometries will be addressed in the future.

TABLE I

VALUES OF $N_{e}^{\phi, s}, N_{\text {act }}^{\phi, s}$ AND $N_{c}$ OF AN ELLIPTICAL ARC FOR DIFFERENT VALUES OF $e$ AND $\alpha$ WHEN $p=10 \lambda$.

\begin{tabular}{|c|c|c|c|c|c|}
\hline \multicolumn{6}{|c|}{$\alpha=\pi / 8$} \\
\hline$e$ & $N_{e}^{\phi}$ & $N_{\text {act }}^{\phi}$ & $N_{e}^{S}$ & $N_{a c t}^{S}$ & $N_{c}$ \\
\hline 0.1 & 15 & 15 & 15 & 15 & 45 \\
\hline 0.3 & 13 & 13 & 13 & 13 & 37 \\
\hline 0.5 & 11 & 11 & 11 & 11 & 33 \\
\hline 0.7 & 9 & 10 & 9 & 10 & 29 \\
\hline 0.9 & 9 & 9 & 9 & 9 & 27 \\
\hline \multicolumn{6}{|c|}{$\alpha=\pi / 4$} \\
\hline$e$ & $N_{e}^{\phi}$ & $N_{\text {act }}^{\phi}$ & $N_{e}^{S}$ & $N_{a c t}^{s}$ & $N_{c}$ \\
\hline 0.1 & 31 & 29 & 29 & 29 & 83 \\
\hline 0.3 & 27 & 26 & 27 & 25 & 73 \\
\hline 0.5 & 25 & 23 & 23 & 23 & 65 \\
\hline 0.7 & 23 & 20 & 21 & 20 & 59 \\
\hline 0.9 & 23 & 19 & 19 & 18 & 55 \\
\hline \multicolumn{6}{|c|}{$\alpha=3 \pi / 8$} \\
\hline$e$ & $N_{e}^{\phi}$ & $N_{\text {act }}^{\phi}$ & $N_{e}^{S}$ & $N_{a c t}^{S}$ & $N_{c}$ \\
\hline 0.1 & 49 & 43 & 47 & 46 & 111 \\
\hline 0.3 & 49 & 40 & 43 & 40 & 105 \\
\hline 0.5 & 49 & 36 & 41 & 36 & 97 \\
\hline 0.7 & 49 & 33 & 39 & 33 & 91 \\
\hline 0.9 & 49 & 31 & 35 & 30 & 87 \\
\hline \multicolumn{6}{|c|}{$\alpha=\pi / 2$} \\
\hline$e$ & $N_{e}^{\phi}$ & $N_{\text {act }}^{\phi}$ & $N_{e}^{S}$ & $N_{a c t}^{S}$ & $N_{c}$ \\
\hline 0.1 & 69 & 62 & 65 & 62 & 125 \\
\hline 0.3 & 81 & 57 & 67 & 57 & 125 \\
\hline 0.5 & 95 & 53 & 67 & 53 & 125 \\
\hline 0.7 & 107 & 50 & 67 & 50 & 125 \\
\hline 0.9 & 119 & 49 & 65 & 46 & 125 \\
\hline
\end{tabular}

TABLE II

VALUES OF $N_{e}^{\phi, s}, N_{a c t}^{\phi, s}$ AND $N_{\mathrm{C}}$ OF AN ELLIPTICAL ARC FOR DifFERENT VALUES OF $e$ AND $\alpha$ WHEN $p=50 \lambda$.

\begin{tabular}{|c|c|c|c|c|c|}
\hline \multicolumn{6}{|c|}{$\alpha=\pi / 8$} \\
\hline$e$ & $\mathrm{~N}_{\mathrm{e}}^{\phi}$ & $\mathrm{N}_{\mathrm{act}}^{\phi}$ & $\mathrm{N}_{\mathrm{e}}^{\mathrm{s}}$ & $\mathrm{N}_{\mathrm{act}}^{\mathrm{s}}$ & $\mathrm{N}_{\mathrm{c}}$ \\
\hline 0.1 & 73 & 72 & 73 & 72 & 221 \\
\hline 0.3 & 63 & 62 & 61 & 62 & 189 \\
\hline 0.5 & 55 & 54 & 53 & 54 & 165 \\
\hline 0.7 & 49 & 48 & 47 & 48 & 147 \\
\hline 0.9 & 45 & 43 & 43 & 43 & 131 \\
\hline \multicolumn{6}{|c|}{$\alpha=\pi / 4$} \\
\hline$e$ & $\mathrm{~N}_{\mathrm{e}}^{\phi}$ & $\mathrm{N}_{\mathrm{act}}^{\phi}$ & $\mathrm{N}_{\mathrm{e}}^{\mathrm{s}}$ & $\mathrm{N}_{\mathrm{act}}^{\mathrm{s}}$ & $\mathrm{N}_{\mathrm{c}}$ \\
\hline 0.1 & 151 & 144 & 147 & 144 & 415 \\
\hline 0.3 & 139 & 126 & 131 & 126 & 367 \\
\hline 0.5 & 129 & 111 & 117 & 111 & 329 \\
\hline 0.7 & 119 & 100 & 107 & 99 & 297 \\
\hline 0.9 & 111 & 91 & 97 & 90 & 271 \\
\hline \multicolumn{6}{|c|}{$\alpha=3 \pi / 8$} \\
\hline$e$ & $\mathrm{~N}_{\mathrm{e}}^{\phi}$ & $\mathrm{N}_{\mathrm{act}}^{\phi}$ & $\mathrm{N}_{\mathrm{e}}^{\mathrm{s}}$ & $\mathrm{N}_{\mathrm{act}}^{\mathrm{s}}$ & $\mathrm{N}_{\mathrm{c}}$ \\
\hline 0.1 & 241 & 196 & 231 & 195 & 559 \\
\hline 0.3 & 247 & 184 & 219 & 181 & 521 \\
\hline 0.5 & 249 & 176 & 205 & 171 & 487 \\
\hline 0.7 & 249 & 162 & 191 & 160 & 457 \\
\hline 0.9 & 247 & 151 & 179 & 150 & 431 \\
\hline \multicolumn{6}{|c|}{$\alpha=\pi / 2$} \\
\hline$e$ & $\mathrm{~N}_{\mathrm{e}}^{\phi}$ & $\mathrm{N}_{\mathrm{act}}^{\phi}$ & $\mathrm{N}_{\mathrm{e}}^{\mathrm{s}}$ & $\mathrm{N}_{\mathrm{act}}^{\mathrm{S}}$ & $\mathrm{N}_{\mathrm{c}}$ \\
\hline 0.1 & 345 & 295 & 325 & 299 & 629 \\
\hline 0.3 & 409 & 269 & 337 & 276 & 629 \\
\hline 0.5 & 471 & 251 & 339 & 257 & 629 \\
\hline 0.7 & 535 & 243 & 337 & 245 & 629 \\
\hline 0.9 & 597 & 237 & 329 & 237 & 629 \\
\hline
\end{tabular}

TABLE III

VALUES OF $N_{p}^{\Phi, s}, N_{a c t}^{\Phi, s}$ AND $N_{\mathrm{C}}$ OF A PARABOLIC ARC FOR DIFFERENT VALUES OF $p$ AND $\alpha$.

\begin{tabular}{|c|c|c|c|c|c|}
\hline \multicolumn{6}{|c|}{$\alpha=\pi / 8$} \\
\hline$p$ & $\mathrm{~N}_{\mathrm{p}}^{\phi}$ & $\mathrm{N}_{\mathrm{act}}^{\phi}$ & $\mathrm{N}_{\mathrm{p}}^{\mathrm{s}}$ & $\mathrm{N}_{\mathrm{act}}^{\mathrm{S}}$ & $\mathrm{N}_{\mathrm{c}}$ \\
\hline $10 \lambda$ & 9 & 8 & 9 & 8 & 25 \\
\hline $30 \lambda$ & 25 & 25 & 25 & 24 & 75 \\
\hline $50 \lambda$ & 41 & 41 & 41 & 41 & 125 \\
\hline $80 \lambda$ & 67 & 65 & 65 & 65 & 199 \\
\hline $100 \lambda$ & 83 & 81 & 81 & 81 & 249 \\
\hline \multicolumn{6}{|c|}{$\alpha=\pi / 4$} \\
\hline$p$ & $\mathrm{~N}_{\mathrm{p}}^{\phi}$ & $\mathrm{N}_{\mathrm{act}}^{\phi}$ & $\mathrm{N}_{\mathrm{p}}^{\mathrm{s}}$ & $\mathrm{N}_{\mathrm{act}}^{\mathrm{S}}$ & $\mathrm{N}_{\mathrm{c}}$ \\
\hline $10 \lambda$ & 19 & 18 & 17 & 17 & 53 \\
\hline $30 \lambda$ & 59 & 52 & 51 & 52 & 157 \\
\hline $50 \lambda$ & 99 & 87 & 85 & 86 & 261 \\
\hline $80 \lambda$ & 159 & 138 & 137 & 138 & 417 \\
\hline $100 \lambda$ & 199 & 172 & 171 & 172 & 521 \\
\hline \multicolumn{6}{|c|}{$\alpha=3 \pi / 8$} \\
\hline$p$ & $\mathrm{~N}_{\mathrm{p}}^{\phi}$ & $\mathrm{N}_{\mathrm{act}}^{\phi}$ & $\mathrm{N}_{\mathrm{p}}^{\mathrm{s}}$ & $\mathrm{N}_{\mathrm{act}}^{\mathrm{s}}$ & $\mathrm{N}_{\mathrm{c}}$ \\
\hline $10 \lambda$ & 41 & 30 & 29 & 29 & 83 \\
\hline $30 \lambda$ & 123 & 88 & 85 & 87 & 251 \\
\hline $50 \lambda$ & 205 & 146 & 143 & 145 & 419 \\
\hline $80 \lambda$ & 327 & 232 & 229 & 231 & 671 \\
\hline $100 \lambda$ & 409 & 289 & 289 & 288 & 839 \\
\hline \multicolumn{6}{|c|}{$\alpha=\pi / 2$} \\
\hline$p$ & $\mathrm{~N}_{\mathrm{p}}^{\phi}$ & $\mathrm{N}_{\mathrm{act}}^{\phi}$ & $\mathrm{N}_{\mathrm{p}}^{\mathrm{s}}$ & $\mathrm{N}_{\mathrm{act}}^{\mathrm{S}}$ & $\mathrm{N}_{\mathrm{c}}$ \\
\hline $10 \lambda$ & 89 & 48 & 45 & 44 & 125 \\
\hline $30 \lambda$ & 267 & 141 & 137 & 132 & 377 \\
\hline $50 \lambda$ & 445 & 233 & 229 & 218 & 629 \\
\hline $80 \lambda$ & 711 & 372 & 367 & 350 & 1005 \\
\hline $100 \lambda$ & 889 & 464 & 459 & 436 & 1257 \\
\hline
\end{tabular}


TABLE IV

MAXIMUM DIRECTIONS OF THE ACTUAL FIELD FOR A RECTIINEAR, ElLIPTICAL, PARABOLIC AND SEMI-CIRCUMFERENCE SOURCE.

\begin{tabular}{cc}
\hline \hline Source geometry & $\tilde{\theta}_{\max }$ \\
\hline Linear source & $1.31 \mathrm{rad}$ \\
Elliptical arc & $1.40 \mathrm{rad}$ \\
Parabolic arc & $1.39 \mathrm{rad}$ \\
Semi-circumference & $1.42 \mathrm{rad}$ \\
\hline \hline
\end{tabular}

TABLE V

RATIO BETWEEN THE MAXIMUM AMPLITUdE OF THE PROJECTED FIELD AND ITS VALUE AT THE HORIZON FOR A RECTILINEAR, ELLIPTICAL, PARABOLIC AND SEMI-CIRCUMFERENCE SOURCE.

\begin{tabular}{cc}
\hline \hline Source geometry & $\frac{\left|\tilde{E}\left(\tilde{\theta}_{\max }\right)\right|}{|\tilde{E}(\theta=\pi / 2)|}$ \\
\hline Linear source & $4.72 \mathrm{~dB}$ \\
Elliptical arc & $19.9 \mathrm{~dB}$ \\
Parabolic arc & $16.2 \mathrm{~dB}$ \\
Semi-circumference & $27.5 \mathrm{~dB}$ \\
\hline \hline
\end{tabular}

TABLE VI

$D\left(\theta_{0}\right)$ FOR A RECTILINEAR, ELLIPTICAL, PARABOLIC AND SEMICirCumFERENCE SOURCE, VARYING THE BEAM MAXIMUM ANGLE $\theta_{0}$

\begin{tabular}{cccc}
$\begin{array}{c}\text { Source } \\
\text { geometry }\end{array}$ & $D\left(\theta_{0}=0 \mathrm{rad}\right)$ & $D\left(\theta_{0}=0.7 \mathrm{rad}\right)$ & $D\left(\theta_{0}=1.13 \mathrm{rad}\right)$ \\
\hline Lin. source & $25.5 \mathrm{~dB}$ & $25.0 \mathrm{~dB}$ & $20.6 \mathrm{~dB}$ \\
Ellipt. arc & $27.0 \mathrm{~dB}$ & $26.0 \mathrm{~dB}$ & $24.5 \mathrm{~dB}$ \\
Parab. arc & $26.1 \mathrm{~dB}$ & $26.1 \mathrm{~dB}$ & $22.9 \mathrm{~dB}$ \\
Semi-circ. & $30.2 \mathrm{~dB}$ & $30.6 \mathrm{~dB}$ & $30.5 \mathrm{~dB}$ \\
\hline
\end{tabular}

\section{APPENDIX A}

Hereafter we provide the analytical details of the results of Section III about the upper bounds of the NDF.

\section{A, Angular parametrization: elliptic arc}

For an elliptic arc, with one focus at the origin, the radial coordinate writes

$$
r(\phi)=\frac{p}{1+e \cos \phi}
$$

where $p$ represents the semi-latus rectum and $0<e<1$ the eccentricity, and the norm of the derivative of the curve parametrization is

$$
\left\|\underline{\gamma}^{\prime}(\phi)\right\|=r(\phi) \sqrt{1+\left(\frac{e \sin \phi}{1+e \cos \phi}\right)^{2}}
$$

Then, the phase function of (6) reads as

$$
g(\phi)=\frac{\cos (\theta-\phi)}{1+e \cos \phi}+\frac{m \pi}{\alpha \beta p} \phi
$$

and the stationary point $\phi_{s}$, obtained by imposing

$$
g^{\prime}\left(\phi_{S}\right)=\frac{\sin \left(\theta-\phi_{S}\right)+e \sin \theta}{\left(1+e \cos \phi_{S}\right)^{2}}+\frac{m \pi}{\alpha \beta p}=0
$$

occurs within the integration interval as long as

$$
|m|<\frac{\alpha \beta p}{\pi} \frac{(1+e)}{(1+e \cos \alpha)^{2}}
$$

This constraint is deduced from (A4) if we remember that $\theta \in[-\pi, \pi]$ (meaning that both $\sin \left(\theta-\phi_{s}\right)$ and $\sin \theta$ are at most equal to 1$)$, and that $\phi \in[-\alpha, \alpha]$ with $\alpha \in[-\pi / 2, \pi / 2]$ (implying that the minimum value of the denominator occurs for $\cos (\phi)=\cos \alpha$ ). For $m$ outside the interval (A5), the solution $\phi_{s}$ of (A4) could be complex, leading to a complex $g(\phi)$ and to an exponentially vanishing contribution of the asymptotic expression of (6). Accordingly, the maximum number of Fourier harmonics providing a significant contribution to the far field is $N_{e}^{\phi}=2 M+1$ with

$M=\left[\frac{\alpha \beta p}{\pi} \frac{(1+e)}{(1+e \cos \alpha)^{2}}\right]$

\section{B, Angular parametrization: parabolic arc}

In this case, with the focus at the origin, the radial coordinate is

$$
r(\phi)=\frac{p}{1+\cos \phi}
$$

with $p$ representing the semi-latus rectum, i.e. twice the parabola focal length, while the norm of the derivative of the curve parametrization is

$\left\|\underline{\gamma}^{\prime}(\phi)\right\|=r(\phi) \sqrt{1+\left(\frac{\sin \phi}{1+\cos \phi}\right)^{2}}$

In (6), the phase function reads as

$g(\phi)=\frac{\cos (\theta-\phi)}{1+\cos \phi}+\frac{m \pi}{\alpha \beta p} \phi$

As before, in order to determine the indices' interval where the stationary phase point occurs, we are interested in the first derivative of function (A9), that is,

$g^{\prime}(\phi)=\frac{\sin (\theta-\phi)+\sin \theta}{(1+\cos \phi)^{2}}+\frac{m \pi}{\alpha \beta p}$

By the application of the sum-to-product identity and the half-angle formula, respectively, to the numerator and denominator of the first term of (A10), the previous expression becomes

$g^{\prime}(\phi)=\frac{2 \sin \left(\theta-\frac{\phi}{2}\right) \cos \frac{\phi}{2}}{4 \cos ^{4} \frac{\phi}{2}}+\frac{m \pi}{\alpha \beta p}$

which finally leads to 
$g^{\prime}(\phi)=\frac{\sin \left(\theta-\frac{\phi}{2}\right)}{2 \cos ^{3} \frac{\phi}{2}}+\frac{m \pi}{\alpha \beta p}$

Provided $\beta p \gg 1$, the stationary point $\phi_{s}$ results from the equation

$g^{\prime}\left(\phi_{S}\right)=\frac{\sin \left(\theta-\frac{\phi_{S}}{2}\right)}{2 \cos ^{3} \frac{\phi_{S}}{2}}+\frac{m \pi}{\alpha \beta p}=0$

Since $\sin \left(\theta-\phi_{s} / 2\right)$ is equal to 1 at most and $\cos ^{3}\left(\phi_{s} / 2\right)$ is equal to $\cos ^{3}(\alpha / 2)$ at least when $\theta \in[-\pi, \pi]$ and $\phi \in$ $[-\alpha, \alpha]$, the stationary phase point occurs as long as

$|m|<\frac{\alpha \beta p}{2 \pi} \frac{1}{\cos ^{3}\left(\frac{\alpha}{2}\right)}$.

We can deduce, hence, that the maximum number of Fourier harmonics is $N_{p}^{\phi}=2 M+1$, with

$M=\left[\frac{\alpha \beta p}{2 \pi \cos ^{3}\left(\frac{\alpha}{2}\right)}\right]$.

C Arc length parametrization: elliptic arc

The phase function of (10) is provided by

$g(s)=\frac{\cos (\theta-\phi(s))}{1+e \cos \phi}+\frac{m 2 \pi}{\beta p L} s$

When $\beta p \gg 1$, the main contribution to the integral is due to the stationary phase point $s_{S}$ making it zero the first derivative of the phase function. By applying the chain rule, the condition satisfied by the stationary point can be written as

$\left.\left.\frac{d g}{d \phi}\right|_{\phi\left(s_{S}\right)} \frac{d \phi}{d s}\right|_{s_{S}}=0$

where $\frac{d s}{d \phi}=\left\|\underline{\gamma}^{\prime}(\phi)\right\|$.

Since the source arc must have a length different from zero to be of interest and $\left\|\underline{\gamma}^{\prime}(\phi)\right\|^{-1}>0$ for any $\phi$, to satisfy (A17) $\left.\frac{d g}{d \phi}\right|_{\phi\left(s_{S}\right)}$ must vanish, and, as a result, we obtain the equation

$\left.\frac{d g}{d \phi}\right|_{\phi\left(s_{S}\right)}=\frac{e \sin \theta+\sin \left(\theta-\phi\left(s_{S}\right)\right)}{\left(1+e \cos \phi\left(s_{S}\right)\right)^{2}}+\frac{m 2 \pi}{\beta L} \frac{\sqrt{1+e^{2}+2 e \cos \phi\left(s_{S}\right)}}{\left(1+e \cos \phi\left(s_{S}\right)\right)^{2}}=0$

We can notice that (A18) differs from (A13) only in the second term where, besides the argument of the Fourier exponential (11), a factor due to the derivative of $s(\phi)$ with respect to $\phi$ appears. The maximum allowable harmonic such that the previous condition is satisfied and the stationary phase points belongs to the source domain, is given by

$M=\left[\frac{L}{\lambda} v\right]$

where $v=(1+e) / \sqrt{1+e^{2}+2 e \cos \alpha}$.

\section{Arc length parametrization: parabolic arc}

Turning the attention to the parabolic arc, we first point out that (9) can be evaluated in closed form as

$s(\phi)=-\frac{L}{2}+\frac{p}{2}\left[\ln \left(\frac{\cos ^{2} \frac{\alpha}{2}\left(2 \cos \frac{\phi}{2}+\sin \phi\right)}{\cos ^{2} \frac{\phi}{2}\left(2 \cos \frac{\alpha}{2}-\sin \alpha\right)}\right)+\frac{1}{2}\left(\frac{\sin \phi}{\cos ^{3} \frac{\phi}{2}}+\frac{\sin \alpha}{\cos ^{3} \frac{\alpha}{2}}\right)\right]$

By repeating the same previous steps, we draw the following condition, slightly different from (A13)

$\left.\frac{d g}{d \phi}\right|_{\phi\left(s_{S}\right)}=2 \frac{\sin \left(\theta-\frac{\phi\left(s_{S}\right)}{2}\right) \cos \frac{\phi\left(s_{S}\right)}{2}}{\left(1+\cos \phi\left(s_{S}\right)\right)^{2}}+\frac{m 4 \pi}{\beta L} \frac{\cos \frac{\phi\left(s_{S}\right)}{2}}{\left(1+\cos \phi\left(s_{S}\right)\right)^{2}}=0$

Therefore, the maximum Fourier harmonic order $m$ allowing to a real solution of (A21) is provided by

$M=\left\lceil\frac{L}{\lambda}\right]$

and, consequently, the maximum number of Fourier harmonics is $N_{p}^{s}=2 M+1$. It can be noted that a closed form of the length of a parabolic arc can be obtained as

$L=\frac{p}{2}\left[\frac{\sin \alpha}{\cos ^{3}(\alpha / 2)}+\ln \left(\frac{2 \cos (\alpha / 2)+\sin \alpha}{2 \cos (\alpha / 2)-\sin \alpha}\right)\right]$

\section{APPENDIX B}

The numerical procedure to define the focused, symmetric far field pattern for a semi-circumference source of Section V.B is sketched as follows.

We start from the $P S F_{F F}(\theta, 1.13)$, which is an element belonging to the subspace of the physically realizable far fields.

Next, we consider $E(\theta)=P S F_{F F}(\theta-0.28,1.13)$ and the corresponding physically realizable radiation pattern $\tilde{E}(\theta)$ by (38).

Finally, the far fields assigned in Fig. 13 (solid lines) consist in this latter $\tilde{E}(\theta)$ function centered at three different maximum directions. Note that, strictly speaking, such far fields do not belong to the set of physically realizable radiation patterns, and must be denoted as $E(\theta)$ in accordance with the notation of Section V.

\section{REFERENCES}

[1] L. Josefsson, and P. Persson, Conformal array antenna theory and design. vol. 29, John wiley \& sons, 2006.

[2] Z. Wei, and Y. Junfeng, "A design of vertical polarized conformal antenna and its array based on UAV structure," Int. J. Antenn. Propag., Article ID 9769815, 2017.

[3] H. Xu, J. Cui, J. Duan, B. Zhang, and Y. Tian, "Versatile conical conformal array antenna based on implementation of independent and endfire radiation for UAV applications," IEEE Access, vol. 7, pp. 31207$31217,2019$.

[4] R. Zhou, J. Sun, S. Wei, and J. Wang, "Synthesis of conformal array antenna for hypersonic platform SAR using modified particle swarm optimization," IET Radar Sonar Navig., vol. 11, no. 8, pp. 1235-1242, 2017.

[5] H. Xu, B. Zhang, J. Duan, J. Cui, Y. Xu, Y. Tian, L. Yan, M. Xiong, and Q. Jia, "Wide solid angle beam-switching conical conformal array 
antenna with high gain for 5G applications," IEEE Antennas Wireless Propag. Lett., vol. 17, no. 12, pp. 2304-2308, 2018.

[6] Z. Hao, M. He, and W. Hong, "Design of a millimeter-wave high angle selectivity shaped-beam conformal array antenna using hybrid genetic/space mapping method," IEEE Antennas Wireless Propag. Lett., vol. 15, pp. 1208-1212, 2016.

[7] Z. Zhang, Xu Gao, W. Chen, Z. Feng, and M. F. Iskander, "Study of conformal switchable antenna system on cylindrical surface for isotropic coverage," IEEE Trans. Antennas Propag., vol. 59, no. 3, pp 776-783, 2010.

[8] Y. Xia, B. Muneer, and Qi Zhu, "Design of a full solid angle scanning cylindrical and conical phased array antennas," IEEE Trans. Antennas Propag., vol. 65, no. 9, pp. 4645-4655, 2017.

[9] Y. F. Wu, and Y. J. Cheng, "Proactive conformal antenna array for nearfield beam focusing and steering based on curved substrate integrated waveguide," IEEE Trans. Antennas Propag., vol. 67, no. 4, pp. 23542363, 2019.

[10] W.H. Kummer, "Basic array theory", Proceedings of the IEEE, vol. 80, no. 1, pp 127-140, Jan. 1992.

[11] L. I. Vaskelainen, "Constrained least-squares optimization in conformal array antenna synthesis," IEEE Trans. Antennas Propag., vol. 55, no. 3, pp. 859-867, 2007.

[12] H. Oraizi, and H. Soleimani, "Optimum pattern synthesis of non-uniform spherical arrays using the Euler rotation," IET Microw. Antennas Propag., vol. 9, no. 9, pp. 898-904, 2015.

[13] J. Chen, Y. Ying-Zeng, and J. Yongchang, "A steerable least square approach for pattern synthesis," Prog. Electromagn. Res., vol. 59, pp. 181-191, 2017.

[14] H. Lebret, and S. Boyd, "Antenna array pattern synthesis via convex optimization," IEEE Trans. Signal Process., vol. 45, no. 3, pp. 526-532, 1997.

[15] S. E. Nai, W. Ser, Z. L. Yu, and H. Chen, "Beampattern synthesis for linear and planar arrays with antenna selection by convex optimization," IEEE Trans. Antennas Propag., vol 58, no. 12, pp. 3923-3930, 2010.

[16] B. Fuchs, "Shaped Beam Synthesis of Arbitrary Arrays via Linear Programming", IEEE Antennas and Wireless Propagation Letters, vol. 9, pp. 481-484, May 2010.

[17] O. M. Bucci, G. D'Elia, and G. Romito, "Power synthesis of conformal arrays by a generalised projection method", IEE ProceedingsMicrowaves, Antennas and Propagation, vol. 142, no. 6, pp. 467-451, Dec. 1995.

[18] J. Ou Yang, Q. R. Yuan, F. Yang, H. J. Zhou, Z. P. Nie, and Z. Q. Zhao, "Synthesis of Conformal Phased Array With Improved NSGA-II Algorithm", IEEE Transactions on Antennas and Propagation, vol. 57, no. 12, pp. 4006-4009, Dec. 2009.

[19] W. T. Li, Y. Q. Hei, and X. W. Shi, "Pattern synthesis of conformal arrays by a modified particle swarm optimization", Progress In Electromagnetics Research, vol. 117, pp. 237-252, 2011.

[20] D. W. Boeringer, and D. H. Werner, "Efficiency-constrained particle swarm optimization of a modified bernstein polynomial for conformal array excitation amplitude synthesis", IEEE Transactions on Antennas and Propagation, vol. 53, no. 8, pp. 2662-2673, Aug. 2005.

[21] W. T. Li, X. W. Shi, Y. Q. Hei, S. F. Liu, and J. Zhu, "A Hybrid Optimization Algorithm and Its Application for Conformal Array Pattern Synthesis", IEEE Transactions on Antennas and Propagation,, vol. 58, no. 10 , pp. 3401-3406, Oct. 2010.

[22] A. N. Tikhonov, and V. Y. Arsenin, Solution to ill-posed problems, Winston, 1977.

[23] M. Bertero, and P. Boccacci, Introduction to inverse problems in imaging, CRC press, 1998.

[24] G. Leone, F. Munno, and R. Pierri, "Radiation Properties of Conformal Antennas: The Elliptical Source," Electronics, vol. 8, no. 5, pp. 531, 2019.

[25] G. Toraldo di Francia, "Degrees of freedom of an image," J. Opt. Soc. Am., vol. 59, no. 7, pp. 799-804, 1969.

[26] R. Piestun, and D. A. B. Miller, "Electromagnetic degrees of freedom of an optical system," J. Opt. Soc. Am. A, vol. 17, no. 5, pp. 892-902, 2000.

[27] G. Newsam, and R. Barakat, "Essential dimension as a well-defined number of degrees of freedom of finite-convolution operators appearing in optics," J. Opt. Soc. Am. A, vol. 2, no. 11, pp. 2040-2045, 1985.

[28] R. Pierri, A. Liseno, F. Soldovieri, and R. Solimene, "In-depth resolution for a strip source in the Fresnel zone," J. Opt. Soc. Am. A, vol. 18, no. 2, pp. 352-359, 2001

[29] G. Leone, "Source geometry optimization for hemispherical radiation pattern coverage," IEEE Trans. Antennas Propag., vol. 64, n. 5, pp .2033$2038,2016$.
[30] G. Leone, M.A. Maisto, and R. Pierri, "Application of inverse source reconstruction to conformal antennas synthesis," IEEE Trans. Antennas Propag., vol 66, no. 3, pp. 1436 - 1445, 2018.

[31] G. Leone, M.A. Maisto, and R. Pierri, "Inverse source of circumference geometries: SVD investigation based on Fourier analysis", Prog. Electromagn. Res. M, vol. 76, pp. 217-230, 2018.

[32] F. Gori, and G. Guattari, "Shannon number and degrees of freedom of an image," Opt. Commun., vol. 7, no. 2, pp. 163-165, 1973.

[33] A. J. Den Dekker, and A. Van den Bos, "Resolution: a survey," J. Opt. Soc. Am. A, vol 14, no. 3, pp. 547-557, 1997.

[34] A. J. Devaney, Mathematical foundations of imaging, tomography and wavefield inversion, Cambridge University Press, 2012.

[35] D. K. Barton, Radar equations for modern radar, Artech House, 2013. 